\title{
Probing photospheric magnetic fields with new spectral line pairs
}

\author{
H. N. Smitha ${ }^{1}$ and S. K. Solanki ${ }^{1,2}$ \\ 1 Max-Planck-Institut für Sonnensystemforschung, Justus-von-Liebig-Weg 3, 37077 Göttingen, Germany \\ e-mail: smi tha@mps.mpg.de \\ 2 School of Space Research, Kyung Hee University, Yongin, Gyeonggi 446-701, Republic of Korea
}

Received 29 May 2017 / Accepted 26 September 2017

\section{ABSTRACT}

\begin{abstract}
Context. The magnetic line ratio (MLR) method has been extensively used in the measurement of photospheric magnetic field strength. It was devised for the neutral iron line pair at 5247.1 and $5250.2 \AA$ (5250 $\mathrm{A}$ pair). Other line pairs as well-suited as this pair have not been reported in the literature.

Aims. The aim of the present work is to identify new line pairs useful for the MLR technique and to test their reliability.

Methods. We used a three-dimensional magnetohydrodynamic 3D MHD simulation representing the quiet Sun atmosphere to synthesize the Stokes profiles. Then, we applied the MLR technique to the Stokes $V$ profiles to recover the fields in the MHD cube both at original resolution and after degrading with a point spread function. In both these cases, we aim to empirically represent the field strengths returned by the MLR method in terms of the field strengths in the MHD cube.

Results. We have identified two new line pairs that are very well adapted to be used for MLR measurements. The first pair is in the visible, Fe I 6820-6842 A, whose intensity profiles have previously been used to measure stellar magnetic fields, and the other pair is in the infrared (IR), Fe I $15534-15542 \AA$. The lines in these pairs reproduce the magnetic fields in the MHD cube rather well and, in fact, somewhat better than the original $5250 \AA$ A pair.

Conclusions. The newly identified line pairs complement the old pairs. The lines in the new IR pair, because of their higher Zeeman sensitivity, are ideal for the measurement of weak fields. The new visible pair works best above $300 \mathrm{G}$. The new IR pair, due to its large Stokes $V$ signal samples more fields in the MHD cube than the old IR pair at $1.56 \mu \mathrm{m}$, even in the presence of noise, and hence likely also on the real Sun. Owing to their low formation heights $\left(100-200 \mathrm{~km}\right.$ above $\left.\tau_{5000}=1\right)$, both the new line pairs are well suited for probing magnetic fields in the lower photosphere.
\end{abstract}

Key words. line: profiles - line: formation - Sun: magnetic fields - Sun: photosphere - polarization

\section{Introduction}

Spectral lines offer diagnostics to measure magnetic fields on the Sun. Accurate magnetic field measurement relies on an optimal combination of spectral lines and the method employed to extract the information on the field. Unfortunately, the Stokes profiles $(I, Q, U, V)$ are affected by many other atmospheric parameters besides the field, making the extraction of the field complex and time consuming. To bypass this, at least for the field strength, Stenflo (1973) proposed the magnetic line ratio (MLR) method, which involves determining the intrinsic magnetic field strength $(B)$ from the ratio of Stokes $V$ of two lines. The two spectral lines must form under the same atmospheric conditions but differ in their magnetic sensitivities, given by the effective Landé g-factors $\left(\mathrm{g}_{\text {eff }}\right)$. For weak, height-independent fields, the Stokes $V$ ratio is simply equal to the ratio of their $\mathrm{g}_{\text {eff }}$. In the presence of strong height-independent fields, the ratio saturates and becomes independent of $B$. This method works best for intermediate field strengths where the Stokes $V$ ratio is proportional to $B$ because of the differential Zeeman saturation. Stenflo (1973) applied MLR to the line pair Fe I 5247.1-5250.2 $\AA$ (5250 pair) in the photospheric network, which leads to the discovery of the presence of kilo-Gauss $(\mathrm{kG})$ fields. Since then the MLR method has been widely used to measure photospheric magnetic fields (Stenflo \& Harvey 1985; Solanki et al. 1987; Schüssler \& Solanki 1988; Solanki et al. 1992; Keller et al. 1994; Grossmann-Doerth et al. 1998; Lozitsky et al. 1999; Stenflo 2010, 2011). For reviews on MLR, see Solanki (1993, 2009), de Wijn et al. (2009), Stenflo (2013).
In addition to MLR, line pairs formed at similar heights in the atmosphere but with different $\mathrm{g}_{\text {eff }}$ are used in multi-line inversions to measure magnetic field. Two other spectral line pairs, used for inversions and MLR, are 6301.5-6302.5 (6300 ̊ pair) in the visible (Domínguez Cerdeña et al. 2003a,b; Stenflo 2010; Ishikawa \& Tsuneta 2011; Steiner \& Rezaei $2012)$, and $15648.5-15652.8 \AA(1.56 \mu \mathrm{m}$ pair $)$ in the infrared (IR; e.g. Solanki et al. 1996). The $1.56 \mu \mathrm{m}$ pair was identified by Solanki et al. (1992) and has been used in the measurement of internetwork fields (Lin 1995; Solanki et al. 1996; Khomenko et al. 2003; Martínez González et al. 2007; Lagg et al. 2016). The $6300 \AA$ pair is used to study both quiet and active regions on the Sun (e.g. Domínguez Cerdeña et al. 2003a,b; Socas-Navarro \& Sánchez Almeida 2002, 2003; Socas-Navarro et al. 2004; Martínez González et al. 2006; Centeno et al. 2007; Lites et al. 2008), based on the observations from both ground-based telescopes and the Hinode satellite. The distribution of the quiet Sun photospheric magnetic field revealed by these two line pairs is different, particularly in the internetwork, first observed by Sánchez Almeida et al. (2003). The $1.56 \mu \mathrm{m}$ pair indicates the presence of mostly sub-kG fields while the $6300 \AA$ pair indicates $\mathrm{kG}$ fields. Combined analyses of the two line pairs have been carried out by Socas-Navarro \& Sánchez Almeida (2003), Khomenko et al. (2005), Domínguez Cerdeña et al. (2006), although with contradictory results.

Khomenko \& Collados (2007) applied MLR to the above three line pairs synthesized from a three-dimensional 
Table 1. Atomic parameters of the new and the old line pairs.

\begin{tabular}{|c|c|c|c|c|c|c|c|c|c|}
\hline \multicolumn{10}{|c|}{ New pairs } \\
\hline & Wavelength $(\AA)$ & Element & $J_{1}$ & $J_{\mathrm{u}}$ & $\log (g f)$ & $\chi_{\mathrm{e}}(\mathrm{ev})$ & $\mathrm{g}_{1}$ & $\mathrm{~g}_{\mathrm{u}}$ & $\mathrm{g}_{\text {eff }}$ \\
\hline \multirow{2}{*}{ I } & 6820.3715 & Fe I & 1.0 & 2.0 & -1.32 & 4.63 & 2.5 & 1.83 & 1.5 \\
\hline & 6842.6854 & $\mathrm{Fe} \mathrm{I}$ & 1.0 & 1.0 & -1.32 & 4.63 & 2.5 & 2.5 & 2.5 \\
\hline \multirow{2}{*}{ II } & 6213.4291 & $\mathrm{Fe} \mathrm{I}$ & 1.0 & 1.0 & -2.48 & 2.22 & 2.5 & 1.5 & 2.0 \\
\hline & 6219.2802 & Fe I & 2.0 & 2.0 & -2.43 & 2.19 & 1.83 & 1.5 & 1.67 \\
\hline \multirow{2}{*}{ III } & 15534.257 & $\mathrm{Fe} \mathrm{I}$ & 1.0 & 2.0 & -0.382 & 5.64 & 1.5 & 1.83 & 2.0 \\
\hline & 15542.089 & $\mathrm{Fe} I$ & 1.0 & 0.0 & -0.337 & 5.64 & 1.5 & 0.0 & 1.50 \\
\hline \multicolumn{10}{|c|}{ Old pairs } \\
\hline & Wavelength $(\AA)$ & Element & $J_{1}$ & $J_{\mathrm{u}}$ & $\log (g f)$ & $\chi_{\mathrm{e}}(\mathrm{ev})$ & $\mathrm{g}_{1}$ & $\mathrm{~g}_{\mathrm{u}}$ & $\mathrm{g}_{\text {eff }}$ \\
\hline \multirow{2}{*}{ I } & 5247.0504 & Fe I & 2.0 & 3.0 & -4.946 & 0.087 & 1.5 & 1.75 & 2.0 \\
\hline & 5250.2080 & $\mathrm{Fe} \mathrm{I}$ & 0.0 & 1.0 & -4.938 & 0.121 & 0.0 & 3.0 & 3.0 \\
\hline \multirow{2}{*}{ II } & 6301.5012 & Fe I & 2.0 & 2.0 & -0.718 & 3.654 & 1.83 & 1.5 & 1.67 \\
\hline & 6302.4936 & $\mathrm{Fe} I$ & 1.0 & 0.0 & -1.236 & 3.686 & 2.5 & 0.0 & 2.5 \\
\hline \multirow{2}{*}{ III } & 15648.518 & $\mathrm{Fe} I$ & 1.0 & 1.0 & -0.675 & 5.426 & 3.0 & 3.0 & 3.0 \\
\hline & 15652.874 & $\mathrm{Fe} I$ & 5.0 & 4.0 & -0.043 & 6.246 & 1.51 & 1.49 & 1.53 \\
\hline \multirow{2}{*}{ IV } & 4122.8020 & $\mathrm{Fe} I$ & 2.0 & 3.0 & -1.300 & 2.832 & 1.50 & 1.16 & 0.820 \\
\hline & 8999.5600 & $\mathrm{Fe} I$ & 2.0 & 2.0 & -1.300 & 2.832 & 1.50 & 1.49 & 1.496 \\
\hline
\end{tabular}

Notes. Columns indicate wavelength, ion, multiplet number, total angular momentum quantum number of the lower $\left(J_{1}\right)$ and upper levels $\left(J_{\mathrm{u}}\right)$, the oscillator strength $\log (g f)$, the lower level excitation potential in ev $\left(\chi_{\mathrm{e}}\right)$, Landé g-factors of the lower $\left(\mathrm{g}_{1}\right)$ and upper levels $\left(\mathrm{g}_{\mathrm{u}}\right)$, and the effective Landé g-factor $\left(\mathrm{g}_{\mathrm{eff}}\right)$, respectively.

magnetohydrodynamic (3D MHD) snapshot. They concluded in favour of the $1.56 \mu \mathrm{m}$ and $5250 \AA$ pairs, although they could not recover $\mathrm{kG}$ from the $5250 \AA$ pair. The $6300 \AA$ pair does not reproduce the fields in the MHD cube because of the difference in height of formation (HOF) of the two lines (Shchukina \& Trujillo Bueno 2001; Khomenko \& Collados 2007; Grec et al. 2010). Discrepancies in the results from the $6300 \AA$ pair observations have been reported in Domínguez Cerdeña et al. (2003b), Martínez González et al. (2006). In a contrasting study, Socas-Navarro et al. (2008) concluded that the $6300 \AA$ pair is better than the $5250 \AA$ pair, as they could not recover $\mathrm{kG}$ fields in the network observations from the $5250 \AA$ pair. In Sect. 5 of the present paper, we try to provide an explanation for this discrepancy. In order to compensate for the difference in HOF of the $6300 \AA$ pair, Stenflo (2010), Stenflo et al. (2013) devised a renormalization to the MLR of $6300 \AA$ pair in terms of the $5250 \AA$ pair.

Any differences in the HOF of the lines in a pair increase the difficulty in interpreting the results from the MLR. The formation heights of the lines in $6300 \AA$ pair are separated by more than $100 \mathrm{~km}$ and those in the $1.56 \mu \mathrm{m}$ pair by $\approx 30 \mathrm{~km}$. These issues leave us with a single "ideal" line pair (Stenflo et al. 2013) for the MLR. Socas-Navarro et al. (2007, 2008) proposed that the pair 4122-9000 $\AA$ works better than all the above pairs (i.e. pairs I, II, and III in the lower part of Table 1). However these lines are $5000 \AA$ apart and need to be observed simultaneously. Also, the Landé g-factor of the $4122 \AA$ is low (see Table 1) and hence this line is less sensitive to magnetic fields. A survey of the Fe I lines with different magnetic sensitivities was carried out by Vasilyeva \& Shchukina (2009). These authors present a list of 28 line pairs that are suitable for MLR. However, the lines in most of these pairs are weak and the authors do not discuss their reliability in detail.

After a detailed search in the visible and IR range of the solar spectrum, we identified three new line pairs. Two pairs are in the

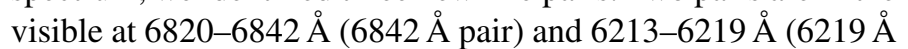

pair). The third pair is in the IR at $15534-15542 \AA(1.55 \mu \mathrm{m}$ pair). The lines in each pair have identical/similar atomic parameters but different $g_{\text {eff }}$. We find that the $6842 \AA$ and $1.55 \mu \mathrm{m}$ pairs are more suitable for MLR than the $6219 \AA$ pair. The lines in these two pairs are formed deep in the photosphere. We compare the performance of the new and old line pairs by applying the MLR method to the Stokes profiles in a 3D MHD cube, and by comparing the results with the fields in the cube. This is carried out at both original resolution of the cube and after applying a degradation. In the first case, we show that the magnetic field strengths given by MLR are best represented when the field strengths in the MHD cube are weighted by the response functions (RFs) of Stokes $V$ profiles and then integrated over the optical depth. In the presence of instrumental degradation, this is very challenging. In this paper, we have made the first attempt to empirically represent the magnetic field strengths returned by the MLR method in a realistic atmosphere with realistic degradation.

In Sect. 2, we discuss the atomic parameters of the new lines. In Sect. 3, we compute their HOF from the response functions. A detailed comparison between the $B$ from the MLR and MHD cube is presented in Sect. 4. In Sect. 5, we repeat the analyses by spatially and spectrally degrading the Stokes profiles and present the conclusions in Sect. 6.

\section{Atomic parameters}

The new and old line pairs are listed in Table 1. The atomic parameters are taken from Kurucz ${ }^{1}$, NIST ${ }^{2}$, and VALD $^{3}$ atomic databases.

The newly identified pairs were listed in Solanki \& Stenflo (1985), Solanki et al. (1992), Ramsauer et al. (1995). The intensity profiles of the $6842 \AA$ line pair was used earlier by Rüedi et al. (1997) to measure the magnetic fields on cool dwarfs

\footnotetext{
http: //kurucz . harvard. edu/linelists.html

http://www.nist.gov/pml/data/asd.cfm

http://vald.astro.uu.se/
} 
using inversions. Owing to its large $\mathrm{g}_{\text {eff }}$, the $6842 \AA$ line was used by Balthasar \& Schmidt (1993), Wiehr (2000) to study sunspots and filaments. Furthermore, the $6842 \AA$ line in combination with Fe I $6843 \AA$ line was used by Saar et al. (1994) to measure magnetic fields in late-type stars. The two lines in the $6842 \AA$ are separated by $22 \AA$ and are unblended. They have identical oscillator strengths $(\log (g f))$ and excitation potentials $\left(\chi_{\mathrm{e}}\right)$ with very different $g_{\text {eff }}$. In the absence of a magnetic field, the lines are formed at the same height in the atmosphere and further details are discussed in Sect. 3.2. Because of their high excitation potential, these lines are less sensitive to fluctuations in $T$ than the $5250 \AA$ A pair.

The lines in the $6219 \AA$ pair, have the same $\log (g f)$ and nearly same $\chi_{\mathrm{e}}$. Although they are formed at the same height in the atmosphere for $B=0$, their $\mathrm{g}_{\text {eff }}$ differ by only $20 \%$, rendering them non-ideal for MLR. The third new pair is in the IR, separated by $8 \AA$ at $15534-15542 \AA$. They belong to different multiplets but have the same $\chi_{\mathrm{e}}$ and similar $\log (g f)$. The red wing of the $15534 \AA$ line is affected by a minor unidentified blend which, may not significantly affect the Stokes $V$ profiles. The blue wing is clean without any blends. The $15542 \AA$ line has no visible blends in the solar spectrum, however, Solanki et al. (1990) indicate the presence of three $\mathrm{Mg}$ I blends. These three $\mathrm{Mg}$ I lines are not listed in the Kurucz, NIST, or VALD atomic databases and appear to have been present only in older databases, so that they may be spurious. According to Ramsauer et al. (1995) this line is only lightly blended by a Si I line at $15542.016 \AA$.

The new line pairs in the visible and IR are separated by $22 \AA$ and $8 \AA$, respectively. The IR pair can be observed with the GREGOR Infrared Spectrograph (GRIS; Collados et al. 2012) as a spectral range as wide as $20 \AA$ has been observed with this instrument (Lagg et al. 2016). It is possible to cover the $22 \AA$ range of the visible line pair using $2 k \times 2 k$ detector at a spectral resolving power of 270000 or better. The new line pairs can be observed with the spectro-polarimeters at the upcoming Daniel K. Inouye Solar Telescope (DKIST) such as the Visible SpectroPolarimeter (ViSP; Elmore et al. 2014) and the Diffraction Limited Near Infrared Spectro-Polarimter (DL-NIRSP; Elmore et al. 2014).

\section{Height of formation}

\subsection{Three-dimensional MHD cube and profile synthesis}

We use a snapshot of a 3D MHD simulation computed from the MURaM code (Vögler et al. 2005). We have selected a cube from the set used by Riethmüller et al. (2014) but with a different resolution. The size of the cube is $(6 \times 6 \times 1.4) \mathrm{Mm}$ with a resolution of $(20.83 \times 20.83 \times 14) \mathrm{km}$. The cube has an unsigned average line-of-sight (LOS) magnetic field of $50 \mathrm{G}$. The properties of the cube, such as the temperature $(T)$, LOS velocity $\left(v_{\text {LOS }}\right)$, and $B$ at $\log \left(\tau_{5000}\right)=0$ are shown in Fig. 1 . The cube represents the quiet Sun atmosphere, dominated by weak and intermediate fields. However there are a few patches of strong magnetic fields in the lower right corner and in the middle left, seen in the right panel of Fig. 1. The Stokes profiles are synthesized using the Stokes-Profiles-INversion-O-Routines (SPINOR) of Frutiger et al. (2000), Frutiger (2000) run in its forward mode along each vertical column of the MHD cube (1.5D) at a heliocentric angle of $\mu=1$.

In Fig. 2, we present the Stokes profiles of all the four line pairs spatially averaged over a small region of $0.4^{\prime \prime} \times 0.4^{\prime \prime}$ close to the centre of the analysed MHD snapshot, indicated by the
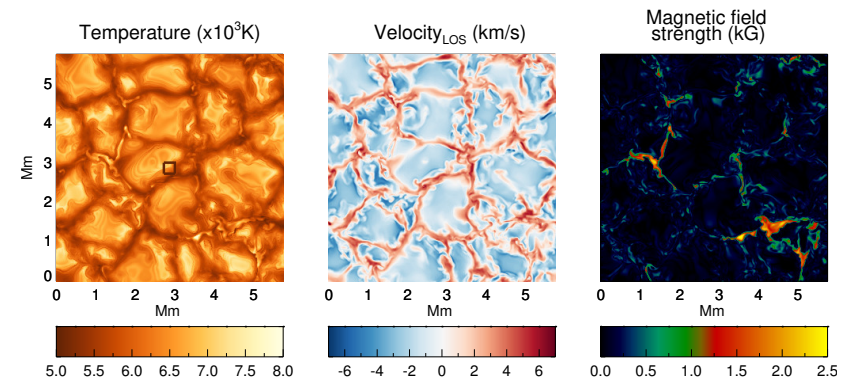

Fig. 1. Temperature, line-of-sight (LOS) velocity, and magnetic field maps of the MURaM MHD cube at $\log \left(\tau_{5000}\right)=0$. The cube has an average unsigned LOS magnetic field of $50 \mathrm{G}$. The black box near the centre of the first panel represents the area over which the Stokes profiles in Fig. 2 are averaged. The size of the box is $0.4^{\prime \prime} \times 0.4^{\prime \prime}$.

black box in the left panel of Fig. 1. The size of the averaged area is chosen to match the resolution of recent observations at the GREGOR telescope (Schmidt et al. 2012) with the GREGOR Infrared Spectrograph (GRIS) instrument (Collados et al. 2012) such as those presented in Lagg et al. (2016).

In the visible range, the new $6842 \AA$ pair is weaker than the $5250 \AA$ pair in both intensity and polarization; we point out the different vertical scales. However, in the IR, although the $15648 \AA$ line of the old IR pair is strong and has large Stokes amplitudes $(Q, U, V)$, the $15652 \AA$ line has much weaker amplitudes, especially in $Q$ and $U$ (see also Martínez González et al. 2008; Lagg et al. 2016) than the lines of the new pair. This makes it harder to use the lines of the old IR pair together, in MLR and in inversions when the profiles are affected by noise. In this respect, the new $1.55 \mu \mathrm{m}$ pair offers a great advantage as both the lines have large Stokes amplitudes. The strong linear polarization signals can be particularly favourable for measuring vector magnetic fields using inversions.

\subsection{Response functions}

To compare the HOF of the line pairs, we use response functions (RFs; Beckers \& Milkey 1975). These functions measure the responses of the line profiles to variations in atmospheric properties such as $T, v_{\mathrm{LOS}}$ and $B$. Using the SPINOR code, we compute the RFs of the Stokes $I$ and $V$ profiles of all the lines to these three atmospheric properties. For the Stokes $I$ profiles, we use the RF at the line centre wavelength, and for Stokes $V$ we use the RF at the wavelength corresponding to the largest peak in the $V$ profile. This is because later, in Sect. 4, we compute the MLR from this largest peak, also referred to as the prominent peak. The HOF is then assumed to be at the centroid of the RFs. The distribution of the HOF across the cube, for different spectral lines, from RFs of Stokes $I$ and $V$ profiles to $T$ are shown in the first two rows of Figs. 3 and 5, respectively. The third row is the unsigned difference in the HOF $(\delta \mathrm{HOF})$ between the lines in the pair. The histograms of the distribution of $\mathrm{HOF}$ and $\delta \mathrm{HOF}$ are shown in the last two rows. The reference height $z=0 \mathrm{~km}$ corresponds to the geometrical layer where $\log \left(\tau_{5000}\right)$, on average, is zero. This HOF represents the atmospheric height that is most sampled by the spectral line. In other words, the Stokes profiles are strongly influenced by the physical conditions at the HOF of the line.

Although $T$ has a dominant influence on the spectral lines and their formation, the RFs from $v_{\mathrm{LOS}}, B$, and magnetic field inclination $(\gamma)$ also provide valuable information, especially for the MLR. Gradients in the $v_{\mathrm{LOS}}, B$, and $\gamma$ affect the shapes of 

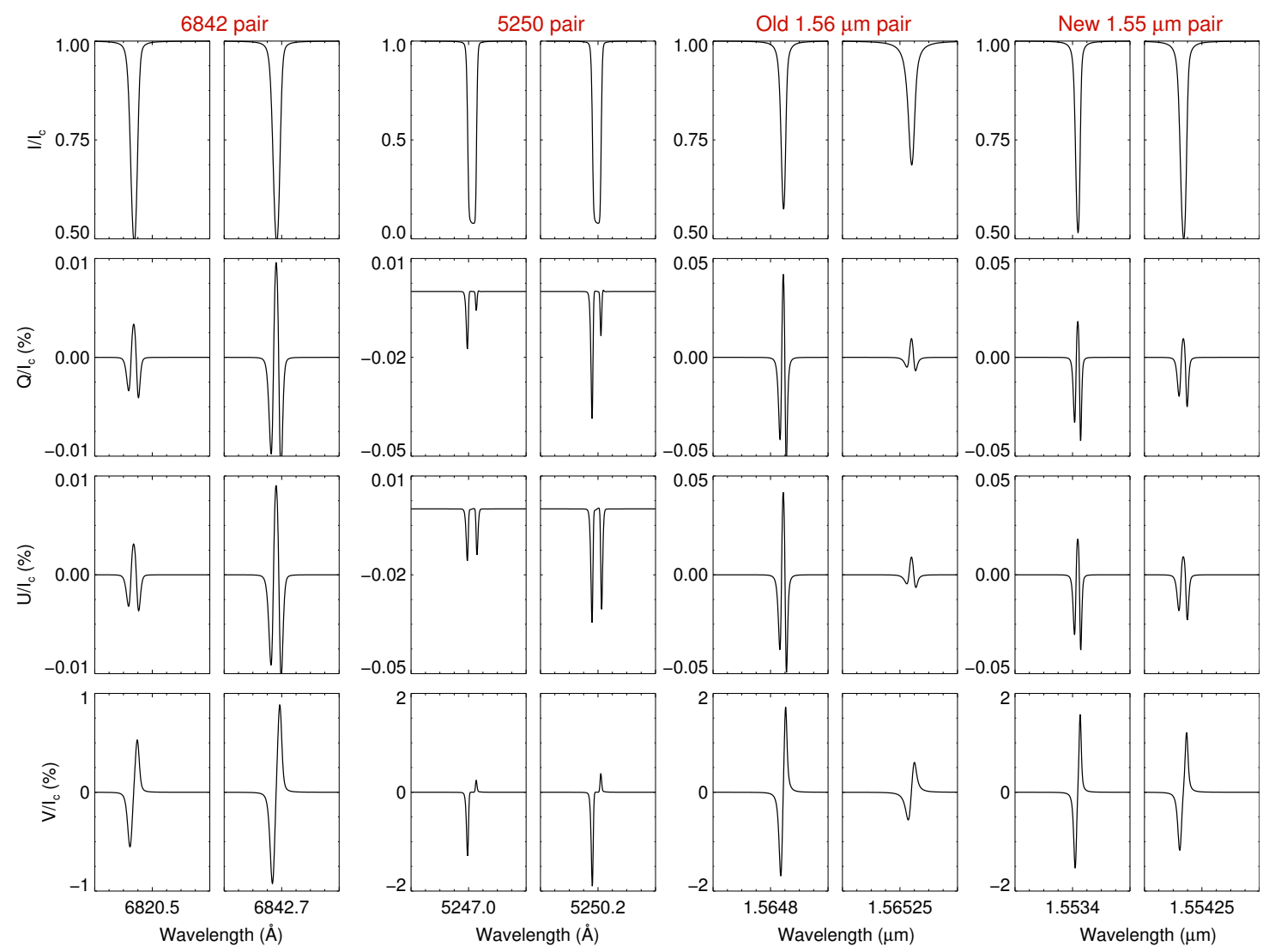

Fig. 2. Stokes profiles of the four line pairs from the MHD cube, averaged over a box of size $0.4^{\prime \prime} \times 0.4^{\prime \prime}$. The location of the box is shown in the first panel of Fig. 1.

the Stokes $V$ profiles, resulting in asymmetries (Khomenko et al. 2005). Hence, the MLR works best if the two lines sample the same $v_{\mathrm{LOS}}, B$, and $\gamma$, in addition to $T$. To confirm this, we computed the $\delta \mathrm{HOF}$ for each line pair from the RFs of Stokes $I$ and $V$ profiles to $v_{\mathrm{LOS}}, B$, and $\gamma \mathrm{RFs}$, in the same way as we did for the $T$ RFs. The variations in $\delta \mathrm{HOF}$ and the histograms are shown in Figs. 4 and 6.

\subsubsection{Old pairs}

From the $T$ RFs of Stokes $I$ (Fig. 3), the $5247 \AA$ and $5250 \AA$ lines are formed at similar heights in the atmosphere for $B=0$ and for weak fields. The histograms of the distribution of HOFs obtained for vertical rays passing through each horizontal pixel of the MHD snapshot for both the lines almost entirely overlap and peak around $300 \mathrm{~km}$ and the $\delta \mathrm{HOF}$ has a narrow spread with a peak at $20 \mathrm{~km}$. They start to differ for intermediate and strong fields where the $\delta \mathrm{HOF}$ can be as high as $100 \mathrm{~km}$. These pixels correspond mostly to the edges of granules to intergranular lanes. In regions of strong magnetic field concentrations (seen at bottom right and middle left of the atmosphere), not only do the HOF of both lines decrease owing to plasma evacuation leading to a drop in the gas pressure, the $\delta \mathrm{HOF}$ also increases owing to the difference in Landé factor. The histograms of the $\delta \mathrm{HOF}$ deduced from $v_{\mathrm{LOS}}, B$, and $\gamma$ RFs of Stokes $I$ profiles at the line centre (Fig. 4), peak close to zero and at a few pixels reach values as high as $150 \mathrm{~km}$ in the strong magnetic regions. Differences in HOF are also seen in the regions surrounding the strong field concentrations because of the magnetic canopies, leading to the measurement of stronger $B$ from MLR (Sect. 4, see also Khomenko \& Collados 2007).
The $T$ RFs of Stokes $I$ profiles (Fig. 3) of the $1.56 \mu \mathrm{m}$ pair indicate that the two lines are most commonly formed around $30 \mathrm{~km}$ apart. However this difference decreases for the $v_{\mathrm{LOS}}, B$, and $\gamma$ RFs (Fig. 4). In particular, the two lines sample similar $B$, despite the difference in their HOFs from the $T$ RFs of Stokes $I$ profiles.

From the Stokes $V$ profiles, since the RFs are considered at the wavelengths of the prominent peak, which is away from the line centre, the HOFs are slightly lower in the atmosphere, especially for the $5250 \AA$ pair (Fig. 5). For the $1.56 \mu \mathrm{m}$ pair, the distribution of the HOFs from Stokes $V$ profiles in Fig. 5 nearly overlap, unlike from the Stokes $I$ profiles (Fig. 3). Other than these difference, the overall distribution of the HOFs and the $\delta \mathrm{HOF}$ from $T, v_{\mathrm{LOS}}, B$, and $\gamma \mathrm{RFs}$ are similar to the case of RFs for Stokes $I$, although in general the difference in the HOFs is now smaller, implying that the MLR should work better than suggested by Figs. 3 and 4 alone.

\subsubsection{New pairs}

Lines in the newly identified $6842 \AA$ pair sample the same heights over most of the atmosphere, evident from the maps of HOFs deduced from the RFs of both Stokes $I$ and $V$ profiles to different atmospheric properties. Like the other pairs, this changes in the strong magnetic regions, with differences in $\mathrm{HOF}>50 \mathrm{~km}$ at some pixels, unavoidably caused by the different Landé factors of the two lines. However, unlike the other line pairs, the $\delta \mathrm{HOF}$ for the new pair always peaks at zero, with a narrow spread in all the different cases shown in Figs. 3-6. This makes the line pair most suitable for the MLR method, of all the considered pairs, at least in this respect. Also, the pair is formed 


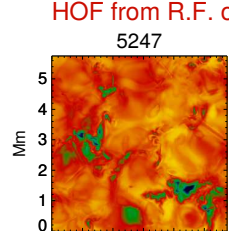

5250

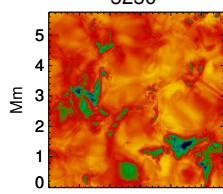

Difference
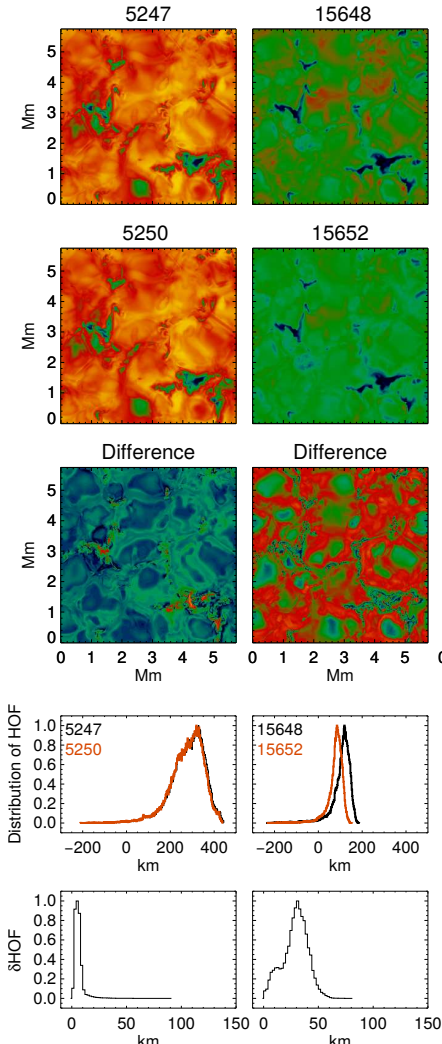

15652

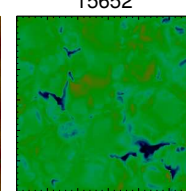

Difference
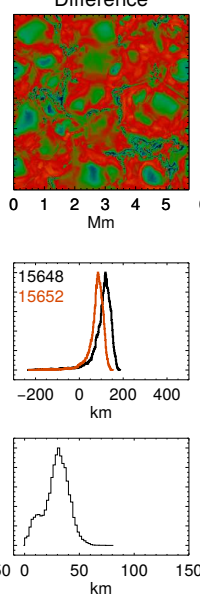

$400-200$

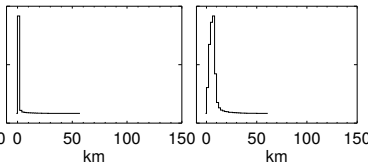

Fig. 3. Maps of height of formation (HOF) for different line pairs deduced from the centroid of the response function (RF) of Stokes $I$ profiles to temperature, at the central wavelength. First and second rows: distribution of HOF referring to individual spatial pixels of the MHD cubes is shown. Third row: difference (absolute) in the HOF is shown. Fourth row: histogram of the HOF of the two lines in each pair is shown Fifth row: histogram of the absolute differences in the HOF is shown.

around $100 \mathrm{~km}$ above $\log \left(\tau_{5000}\right)=0$ and samples deep photospheric layers similar to the old $1.56 \mu \mathrm{m}$ pair. Thus we now have a line pair in the visible, which can be used complementarily with the IR pair to probe the deep photospheric layers. In addition, the spread in the HOF of the $6842 \AA$ pair is small and their individual RFs are quite narrow, implying that they see a narrow range of atmospheric layers due to their higher excitation potentials, unlike the $5250 \AA$ pair. This makes them less sensitive to magnetic field gradients, which is an advantage for the line ratio, but a disadvantage for their use in height-dependent inversions. The case with the new $1.55 \mu \mathrm{m}$ pair is similar. The two lines are formed at the same height, deep in the photosphere, as seen in the maps of HOFs from the RFs of both Stokes $I$ and $V$ profiles (Figs. 3-6). Particularly in the granules, their $\delta \mathrm{HOF}$ is close to zero. Because of the increased Zeeman sensitivity of the IR lines, this pair is well suited for the measurement of weak granular fields, as is discussed in Sect. 4. Also, there is a similarity in HOF between the new IR pair and the $15648 \AA\left(\mathrm{g}_{\text {eff }}=3\right)$ line in the old IR pair. If two wavelength ranges can be covered simultaneously, then the two IR pairs, with their very different magnetic sensitivities, can be used together.

\section{Comparison with three-dimensional MHD simulations}

We define the MLR of a line pair as the ratio of Stokes $V$ amplitude from the magnetically weaker line (smaller $\mathrm{g}_{\mathrm{eff}}$ ) to
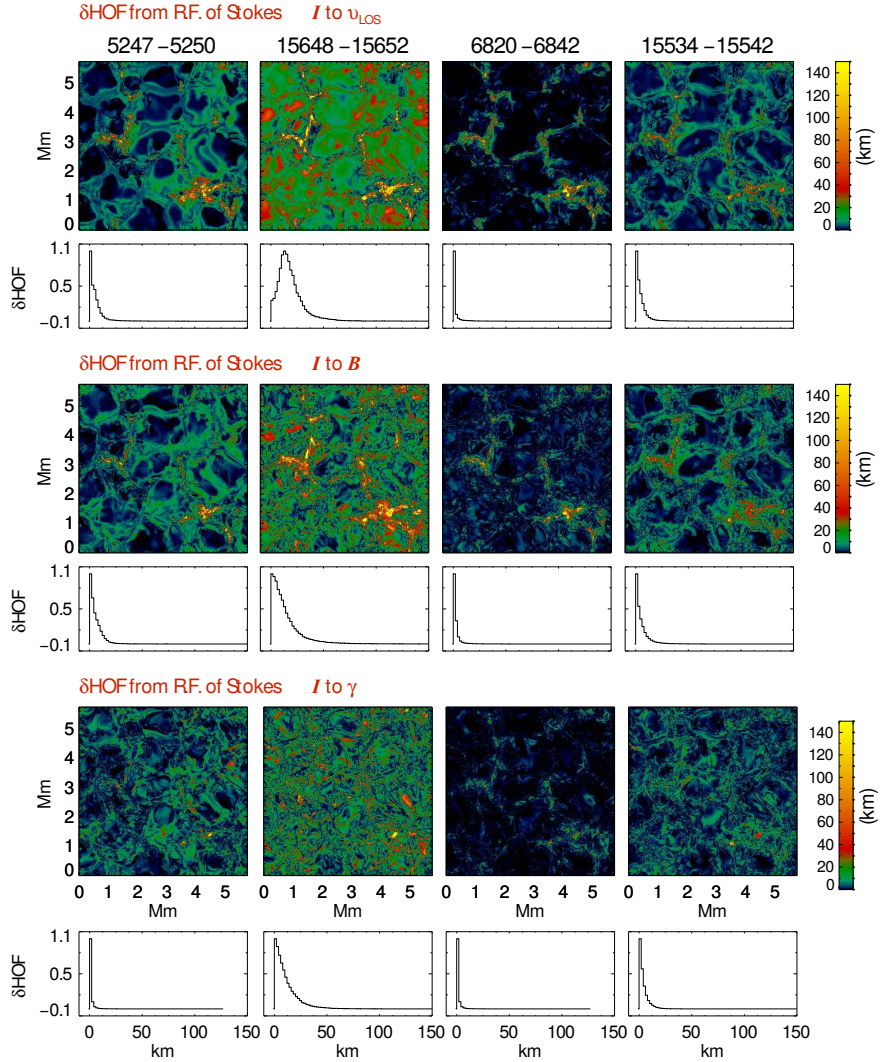

Fig. 4. Maps of difference in heights of formation and their histograms. The maps are constructed similar to the third and fifth rows of Fig. 3, but computed from the RFs of Stokes I profiles to perturbations in velocity, magnetic field strength, and inclination.

the stronger line (higher $\mathrm{g}_{\text {eff }}$ ), similar to Khomenko \& Collados (2007). To extract $B$ from this ratio, we need a calibration curve. Though neither micro- nor macro-turbulent velocities $\left(v_{\text {mic,mac }}\right)$ are used in the computation of the Stokes profiles, they are still broadened by the often strong vertical gradients in $v_{\text {LOS }}$. Hence we must account for the widths of the spectral lines in the construction of the calibration curves. In Fig. 7, we show the distribution of the line widths, defined in this case as the full width at half maximum (FWHM), for lines in the four pairs. Except for the old $1.56 \mu \mathrm{m}$ pair, the lines in each pair have practically the same line widths. The difference in line widths is a product of the difference in HOF between the lines in the $1.56 \mu \mathrm{m}$ pair.

Ideally, before applying the MLR, one must construct calibration curves at every pixel by fitting the intensity profiles using both micro and macro-turbulence for the four line pairs. This increases the number of calibration curves and they are not unique, as different combinations of micro- and macro-turbulence are possible. In order to simplify this, we first set $v_{\text {mac }}=0$ and match the line widths using $v_{\text {mic }}$. We then divide the range of line widths into 10 bins of size $3 \mathrm{~m} \AA$ for the visible pairs and $10 \mathrm{~m} \AA$ for the IR pairs. We vary a height-independent $v_{\text {mic }}$ from 0.0 to $3.5 \mathrm{~km} \mathrm{~s}^{-1}$ to get the required line width and construct a calibration curve for each width bin and each line pair. Figure 8 shows the resulting calibration curves for each line pair. The curves are computed using the HSRA (Gingerich et al. 1971) model atmosphere. Fitting both line width and depth by varying $v_{\text {mac }}$ and $v_{\text {mic }}$ increases the number of calibration curves. Setting $v_{\text {mac }}=0$ is a choice made to minimize the number of calibration curves. Despite this simplification, we recover the magnetic field strengths in the MHD cube relatively well, as discussed below. 


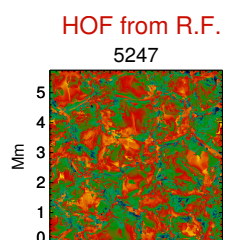

5250

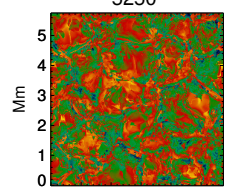

Difference
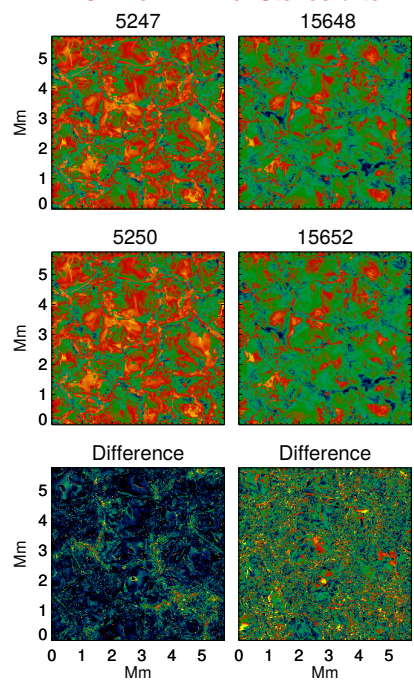

15652

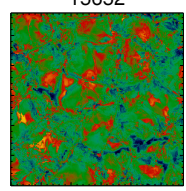

Difference
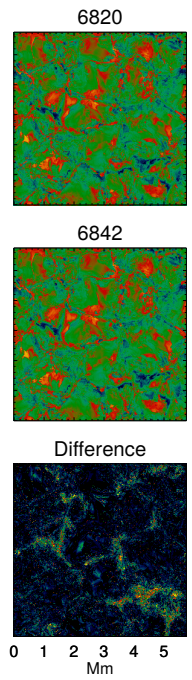

6842

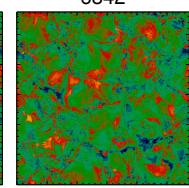

Difference

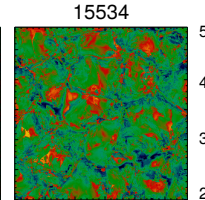

15542

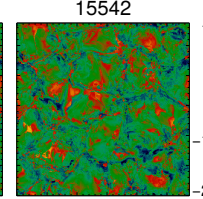

Difference
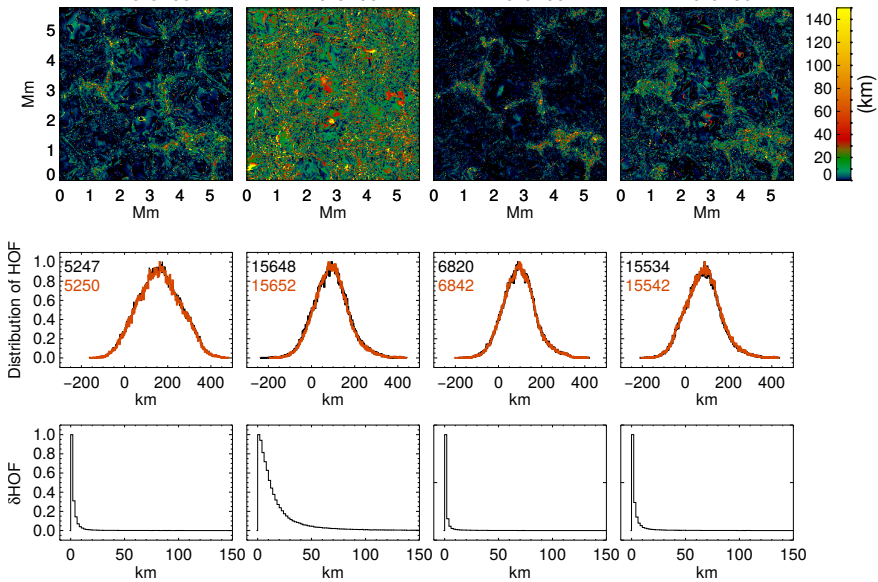

Fig. 5. Maps of height of formation (HOF) for different spectral lines deduced from the centroid of the response function of Stokes $V$ profiles to temperature at the wavelength corresponding to the largest peak in $V$. Different rows represent the same quantities as in Fig. 3.

The calibration curves for the $6842 \AA$ pair, in Fig. 8, starts to decrease below the saturation level once the field strength exceeds a certain threshold value $\left(B_{\mathrm{th}}\right)$. The greater the turbulent velocity or the wider the spectral line, the higher is the value of $B_{\mathrm{th}}$. In the absence of any turbulent velocity (first calibration curve for the $6842 \AA$ pair), $B_{\text {th }} \approx 1200$ G. Similarly, the calibration curves for the new $1.55 \mu \mathrm{m}$ pair continues to increase beyond unity when the field strength exceeds $B_{\text {th }}$ for that pair. This is because of the anomalous Zeeman splitting of the spectral lines. We discuss this in greater detail in the appendix.

The ambiguities involved in the comparison of $B$ from MLR with the 3D MHD cube are more severe than those involved while comparing the inversion results with the MHD simulations. The later case has been discussed in detail in Borrero et al. (2014). For the MLR, a similar comparison is made by Khomenko \& Collados (2007). In this paper, the authors compare the results from MLR with $B$ at $\log \left(\tau_{5000}\right)=-1$ layer in the MHD cube. For only a slice of the cube, they also discuss the comparison with the fields weighted by the response function of Stokes $I$ to $T$. As the lines sample different depths across the cube (Figs. 3-6), comparing the results of MLR with the fields at constant $\tau$ does not properly indicate the reliability of the line pair. Hence, we discuss below a different way of comparing $B$ from MLR and the MHD cube.

Traditionally, the MLR is computed by either taking the ratio of the blue Stokes $V$ peak, as in for example Khomenko \& Collados (2007), Stenflo (2010), Stenflo et al. (2013), or by taking the sum or the average of the blue and red lobes (e.g. Stenflo \& Harvey 1985; Solanki et al. 1987). The rationale behind the former is that the blue peak is less affected by
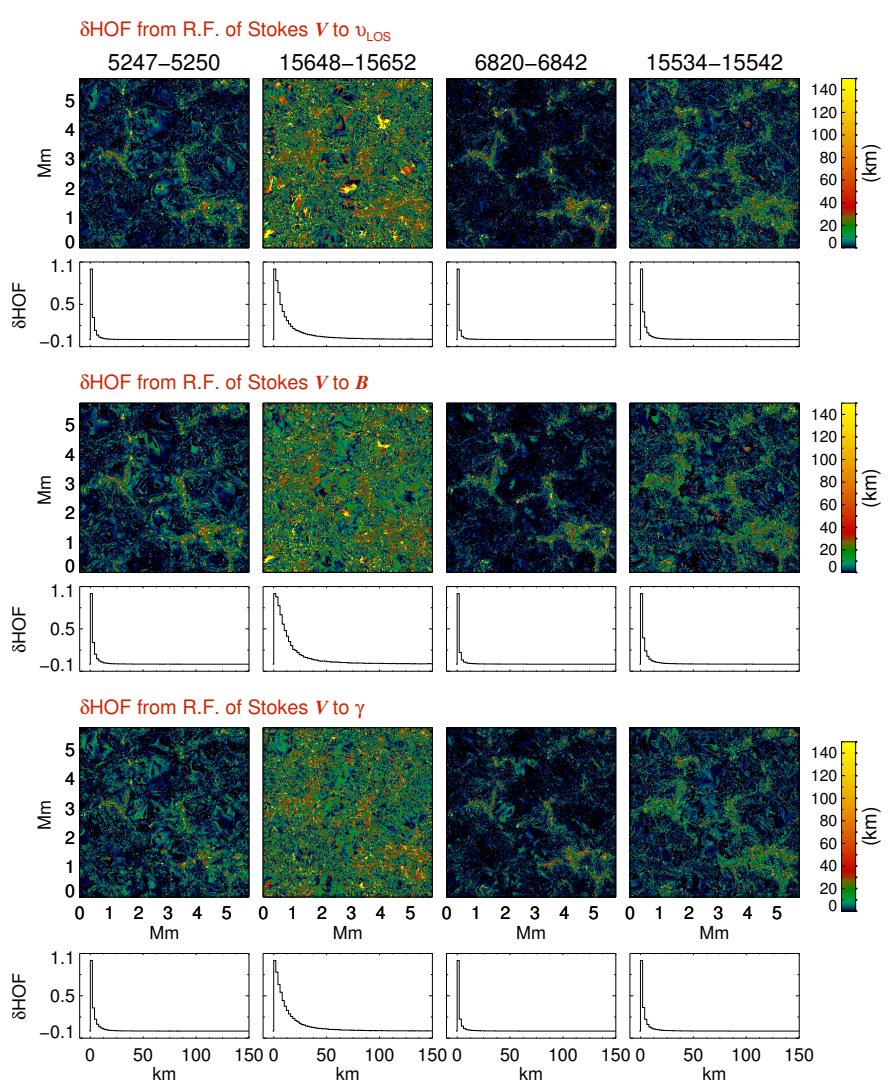

Fig. 6. Maps of difference in heights of formation and their histograms, similar to Fig. 4, but computed from the response functions of Stokes $V$ profiles to perturbations in velocity, magnetic field strength, and inclination.

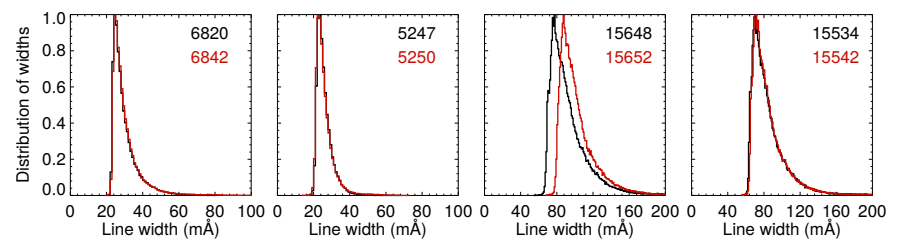

Fig. 7. Distribution of line widths in the four line pairs across the cube.

magnetic and velocity gradients and that they have larger amplitudes (Stenflo 2010). Taking the sum or the average of the blue and red lobes improves the signal-to-noise ratio. However, while comparing with the magnetic field strength in the MHD cube, we find that the ratio of the most prominent peak (the lobe with higher amplitude) in the Stokes $V$ profile performs better than the other two ratios. A similar approach has also been followed by Lagg et al. (2016).

If $B$ from MLR is $B_{\mathrm{MLR}}(x, y)$ and $B$ in the MHD cube is $B_{\mathrm{MHD}}(x, y)$, then

$B_{\mathrm{MHD}}(x, y)=\frac{\int \mathrm{RF} \_\operatorname{tot}_{B}^{V}(x, y, \tau) B(x, y, \tau) \mathrm{d} \tau}{\int \mathrm{RF} \_\operatorname{tot}_{B}^{V}(x, y, \tau) \mathrm{d} \tau}$,

where RF_tot ${ }_{B}^{V}(x, y, \tau)$ is the total RF for the two lines defined as

$\mathrm{RF} \_\operatorname{tot}_{B}^{V}(x, y, \tau)=\mathrm{RF}_{B}^{V}\left(x, y, \tau, \lambda_{p 1}\right)+\mathrm{RF}_{B}^{V}\left(x, y, \tau, \lambda_{p 2}\right)$.

In Eq. (2), $\operatorname{RF}_{B}^{V}\left(x, y, \tau, \lambda_{p 1, p 2}\right)$ are the RFs at wavelengths, $\lambda_{p 1, p 2}$, corresponding to the peak value of the Stokes $V$ profile from the MHD cube. This peak value is then used to compute the MLR. 

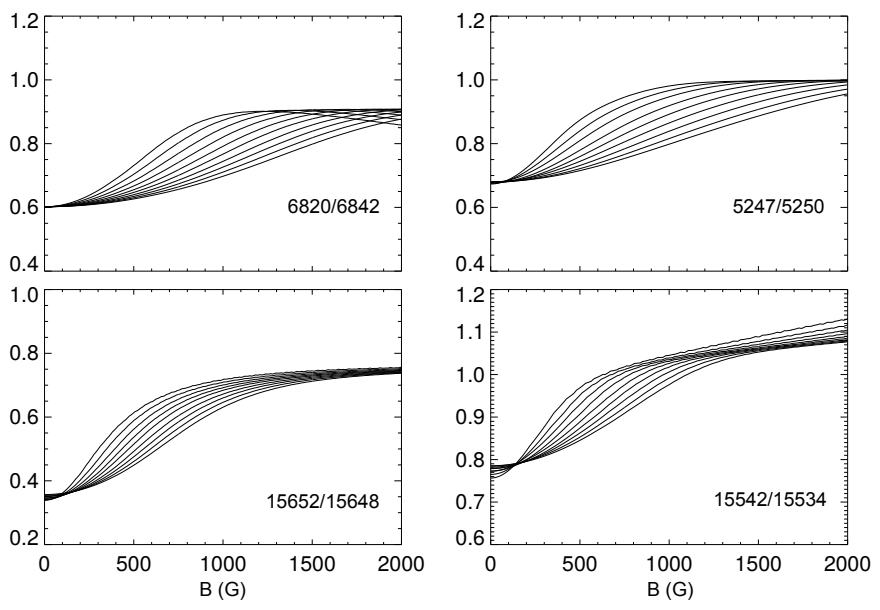

Fig. 8. Ten calibration curves for each line pair, one for each of the 10 bins into which the line widths are divided. The curves are computed for the HSRA model by varying height-independent micro-turbulent velocity from $0.0 \mathrm{~km} \mathrm{~s}^{-1}$ to $3.5 \mathrm{~km} \mathrm{~s}^{-1}$.

In Fig. 9, we show the comparison between $B_{\mathrm{MLR}}$ (first column) and $B_{\mathrm{MHD}}$ (second column) computed using Eqs. (1) and (2). The third column is the difference, $B_{\mathrm{MHD}}-B_{\mathrm{MLR}}$. The last two columns depict the histograms of the differences over the full range of $B_{\mathrm{MHD}}$ (fourth column) and over the range where the MLR method is most effective (fifth column). The latter is plotted starting from the field strengths for which the more Zeeman sensitive line of each pair enters the non-linear Zeeman regime, or in other words, from where the calibration curves start to have a steep gradient. We then apply a Gaussian fit to the histogram and the FWHM of the Gaussian curve is indicated for each pair.

The fields in the MHD cube are well reproduced by all the four line pairs. The differences between the $B_{\mathrm{MLR}}$ and $B_{\mathrm{MHD}}$ seen in the third column resemble the $\delta \mathrm{HOF}$ images in Figs. 3 and 4. When the full range of field strengths are considered, the scatter is the smallest from the $6842 \AA$ pair and largest from the old $1.56 \mu \mathrm{m}$ pair. When the reliability of the pairs are tested over the field strength range where they are most efficient, all the line pairs perform equally well and the scatter is very small.

The difference image in the third column, which covers the full range of field strengths, has contributions from three factors. The first contribution is from those pixels for which the fields are weak and the lines are still in the weak field regime, i.e. the Zeeman splitting is much smaller than the Doppler width. Hence in the fifth column, we show the histogram of the difference by excluding these weak fields. The $6842 \AA$ pair and $5250 \AA$ pair are in this regime up to $\approx 250 \mathrm{G}$. This is seen from the calibration curves in Fig. 8. Here, the Stokes $V$ ratio is equal to the ratio of $\mathrm{g}_{\text {eff }}$ of the two lines. However, the IR pairs are in the weak field regime for field only up to $\approx 100-150 \mathrm{G}$. Hence, they can measure weak granular fields better than the visible pairs. Among the two IR pairs, the new $1.55 \mu$ pair performs even better in the granules because the two lines have the same HOF. This is indicated by the white patches seen in the difference image at the granules.

The second factor contributing to the difference is the increase in $\delta \mathrm{HOF}$ in the regions surrounding strong magnetic field concentrations owing to the canopies (Sect. 3.2.2). From Figs. 3-6, this increase is seen in all the four line pairs. In these regions, the $B_{\mathrm{MLR}}>B_{\mathrm{MHD}}$ and such locations are seen as brown patches surrounding strong field regions in the difference images of Fig. 9, which was also noted by Khomenko \& Collados (2007). Contributions from these pixels to the histogram of the difference (fourth column in Fig. 9) appear in the left wing of the Gaussian, which extends up to $1000 \mathrm{G}$. These pixels do not contribute to the histogram in the fifth column because they are constructed by imposing criteria on $B_{\mathrm{MHD}}$. The $B_{\mathrm{MHD}}$ in these pixels are below the imposed criteria. Differences in $B_{\text {MLR }}$ and $B_{\mathrm{MHD}}$ are also seen along the edges of the granules, i.e along the granular-intergranular boundaries. Once again, this is due to the increase in $\delta \mathrm{HOF}$ caused by strong $T, v_{\mathrm{LOS}}$, and $B$ gradients, seen from Figs. 3-6.

The third factor is the saturation (or near saturation) of the calibration curves for stronger field strengths. The calibration curves for the visible line pairs, for larger line widths, do not saturate even at $2000 \mathrm{G}$ (Fig. 8). For the IR pair, the calibration curves saturate around $1200 \mathrm{G}$.

\section{Magnetic line ratio with degraded profiles}

In the previous section, we discussed the line pairs and MLR under ideal conditions but in reality, the observations from any instrument are affected by noise and atmospheric seeing. In this section we discuss the influence of these effects on the line profiles and the results from MLR.

To simulate the solar observations, we apply both spatial and spectral degradation to the Stokes profiles from the MHD cube and then estimate the $B_{\text {MLR }}$. For this, the synthesized Stokes profiles are convolved with the theoretical point spread function (PSF) of the GREGOR telescope, which includes the effects of spatial stray light. The profiles are then spectrally degraded by convolving them with a Gaussian with $F W H M=30 \mathrm{~m} \AA$ and $100 \mathrm{~m} \AA$, respectively, for the visible and IR line pairs. Later, they are rebinned to a detector pixel resolution of 0 '!2 . For further details on the PSF used, see Lagg et al. (2016).

In addition to the degradation, we add a random noise of $\sigma=1 \times 10^{-3}$ in the units of continuum intensity of the respective pair. We then consider all profiles with an amplitude larger than $3 \sigma$ and apply a median filter over three wavelength pixels to smoothen the Stokes profiles. This threshold is applied to the magnetically weaker of the two lines in the pair. After spectral degradation and filtering, the Stokes profiles are further broadened. Hence we must construct new set of calibration curves for the four pairs. Repeating the same procedure as before, in Fig. 10 we plot the histograms of the line widths over the whole cube. The line widths of the profiles are grouped into bins of $3 \mathrm{~m} \AA$ and $10 \mathrm{~m} \AA$ for the visible and IR pairs, respectively. At first, only $v_{\text {mic }}$ is varied to match the line widths while keeping $v_{\text {mac }}=0$, and the calibration curves are constructed. The effects of $v_{\mathrm{mac}}$ are discussed later in the section.

The MLR estimates $B$ within the resolution element irrespective of the filling factor. In other words, in a resolution element containing a mix of magnetic and nonmagnetic components or strong and weak magnetic components, the MLR measures $B$ mainly from the strong magnetic component in the element and not the spatially averaged $B$ (Stenflo 1973). Hence, to compare with the fields in the MHD cube, we weight $B$ with the $V$ amplitude. By doing so, we give more weight to the magnetic field at locations where the Stokes $V$ profile is stronger. In general these are the stronger magnetic fields (aligned along the line of sight), while the weaker (or more transverse) fields provide a proportionally smaller contribution to the line ratio. When such a weighted magnetic field strength is averaged to match the degraded pixel resolution, the resulting field strength 

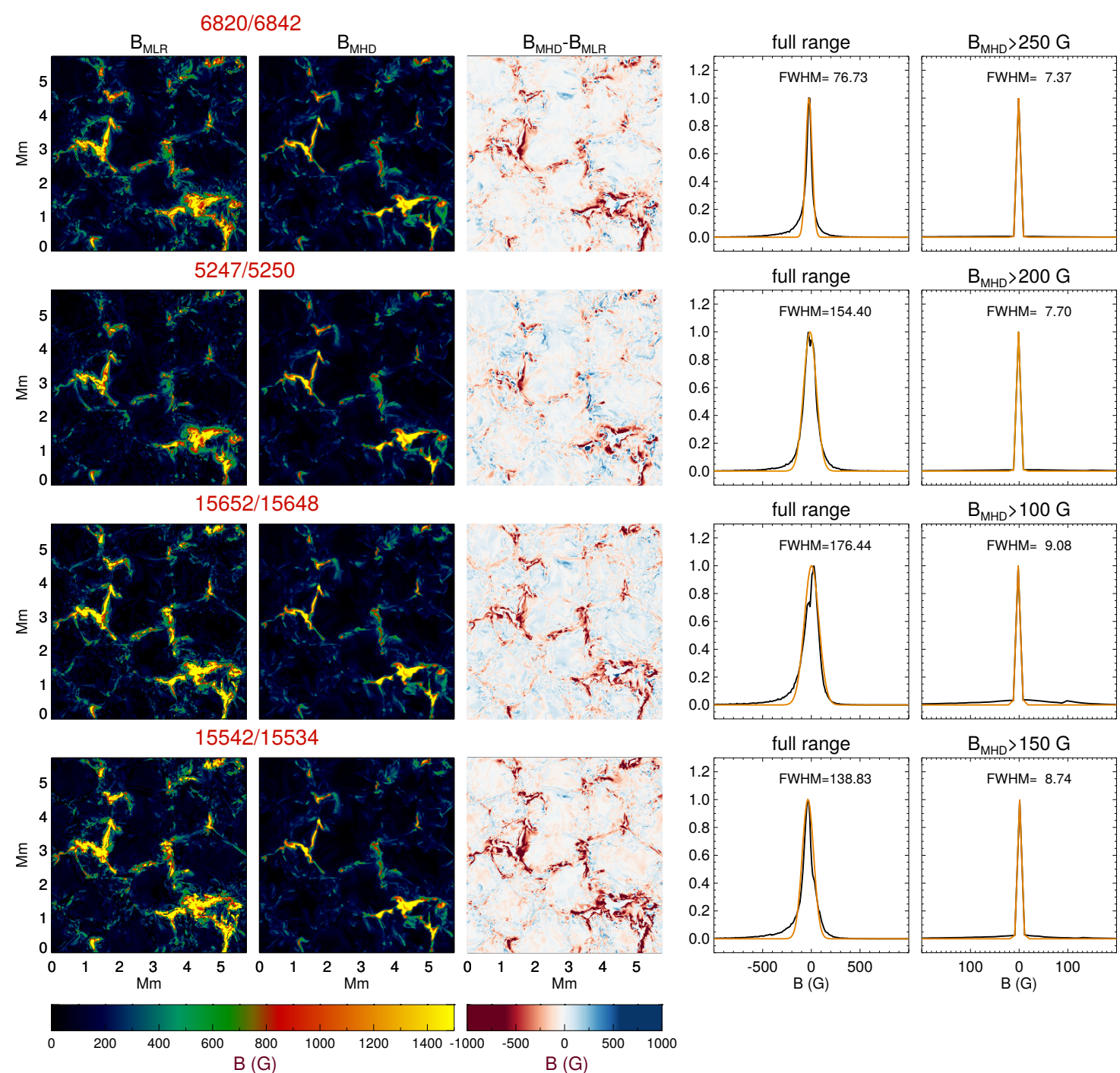

Fig. 9. Comparison between the magnetic field strengths from the MLR $\left(B_{\mathrm{MLR}}\right)$ and the MHD cube $\left(B_{\mathrm{MHD}}\right)$. The fields in the MHD cube are computed using Eqs. (1) and (2). The third column is the difference, $B_{\mathrm{MHD}}-B_{\mathrm{MLR}}$. The fourth column is the histogram of the difference (black curve), which is fit with a Gaussian (brown curve) and the width of the Gaussian is indicated in each case. Fifth column is same as the fourth but only over the field range where the MLR is most effective.

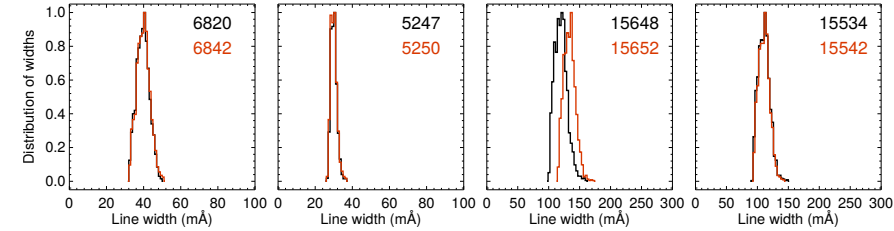

Fig. 10. Distribution of line widths in the four line pairs across the cube after the Stokes profiles are spectrally and spatially degraded.

has contributions mainly from the stronger magnetic component and resemble the field strength measured by MLR. Below we give an empirical relation aiming to provide a magnetic parameter that approximates the field strength sampled by the line ratio technique in the presence of finite spatial resolution. We call the magnetic field computed using this relation as $B_{\mathrm{MHD}-\text { rebin. It is }}$ given by

$$
B_{\text {MHD-rebin }}\left(x_{p}^{\prime}, y_{q}^{\prime}\right)=\frac{\sum_{j=a q} \sum_{i=a p}^{a(j+1)-1}\left[B_{\mathrm{MHD}}\left(x_{i}, y_{j}\right) V\left(x_{i}, y_{j}\right)\right]}{\sum_{j=a q}^{a(j+1)-1} \sum_{i=a p}^{a(i+1)-1}\left[V\left(x_{i}, y_{j}\right)\right]},
$$

where $p=0,1, . ., m-1 ; q=0,1, . ., n-1$, and the summations rebin the quantities in the square brackets. For the present purposes, the rebinning is performed over 7 pixels, i.e. $a=7$, to match a detector pixel resolution of $0 \prime 2$. The dimensions of $B_{\text {MHD-rebin }}\left(x^{\prime}, y^{\prime}\right)$ is $(m, n)$. In the above equation, $B_{\mathrm{MHD}}(x, y)$ is computed from Eqs. (1) and (2). The value $V(x, y)$ is the amplitude of the Stokes $V$ profile at pixel $(x, y)$ from the MHD cube at full resolution. When the fields are weak, $V \propto B$ and from Eq. (3), $B_{\mathrm{MHD} \text {-rebin }}$ is $B_{\mathrm{MHD}}$ averaged over the resolution element.

Figure 12 shows a comparison between the $B_{\mathrm{MLR}}$ from the spatially and spectrally degraded profiles with $B_{\text {MHD-rebin }}$ defined in Eq. (3). In the first row the $B_{\mathrm{MLR}}$ is computed from the calibration curves, which are constructed by varying only $v_{\text {mic }}$ to match the line widths. The maps in the second row are discussed later. The third row shows the magnetic field maps resulting from Eq. (3). The shapes of the magnetic field structures from the MLR method in the first row do not resemble those in the third row. This is because the magnetic field structures in the first row, which are obtained by applying the MLR method on the PSF convolved Stokes $V$ profiles, are smeared out. This effect has not been accounted for in Eq. (3). In order to reproduce 
this effect, we apply the PSF to $B_{\mathrm{MHD}-\text { rebin }}$ to get $B_{\mathrm{MHD}-\text { rebin-PSF }}$ We stress that there is no clear cut physically consistent manner in which $B_{\text {MHD-rebin }}$ can be convolved with the PSF. Our aim here is to empirically get a better idea of what quantity the MLR actually returns in a realistic atmosphere in the presence of spatial smearing. To that end we tried various approaches and compared the resulting maps with the first row of Fig. 12. We found that the best agreement (in the shape of the features) was obtained by

$$
B_{\text {MHD-rebin-PSF }}\left(x^{\prime}, y^{\prime}\right)=\operatorname{PSF}\left(x^{\prime}, y^{\prime}\right) * B_{\text {MHD-rebin }}\left(x^{\prime}, y^{\prime}\right) \text {, }
$$

where $B_{\mathrm{MHD}-\text { rebin }}\left(x^{\prime}, y^{\prime}\right)$ is defined in Eq. (3) and $*$ represents convolution. After applying the PSF, however, the magnetic field is smeared and diluted (Lagg et al. 2016). Thus $B_{\text {MHD-rebin-PSF }}$ is much smaller than $B_{\text {MHD-rebin }}$ (third row of Fig. 12). In the presence of spatial smearing, although result from MLR is spatially smeared, the strength of the field is maintained (i.e. still the intrinsic field strength is reached at the centres of magnetic

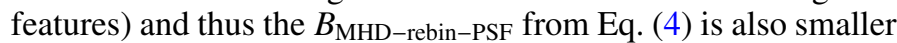
than the MLR results shown in the top row of Fig. 12. Hence we normalize $B_{\mathrm{MHD}-\text { rebin-PSF}}$, such that its maximum field strength matches with the maximum of $B_{\mathrm{MHD}-\text { rebin. In the fourth row }}$ we show maps of the normalized $B_{\text {MHD-rebin-PSF }}$ and the pixels where the degraded Stokes $V$ is smaller than $3 \sigma$ are filtered out. Now the field structures in the first row resemble those in the fourth row.

The $B_{\mathrm{MHD}}$ in Eq. (3) is obtained after weighting the original field in the MHD cube with the response function and integrating over tau, from Eqs. (1) and (2). Therefore, the original intrinsic field strength in the MHD cube is maintained. With Eqs. (3) and (4), we are trying to empirically represent the quantity that MLR method provides in a realistic atmosphere and for realistic instrumental degradation. This is not straightforward and has not been reported in the literature. By comparing the maps in the first and fourth rows in Fig. 12, we see that this empirical representation provides a reasonably close match with $B_{\mathrm{MLR}}$.

Owing to smaller $V$ amplitudes in the $6842 \AA$ line pair and the $1.56 \mu \mathrm{m}$ line pair, about $30-45 \%$ of the profiles are above the $3 \sigma$ threshold. As the lines in the $5250 \AA$ pair and the $1.55 \mu \mathrm{m}$ pair are stronger, more than $80 \%$ of the profiles remain above the $3 \sigma$ level. The $6842 \AA$ pair and the two IR pairs clearly show the presence of $\mathrm{kG}$ fields in the cube. But they are spread over larger areas because of the convolution with the PSF. The green patches surrounding the strong field yellow patches are due to redistribution of the photons caused by the PSF. This is also discussed in detail by Lagg et al. (2016).

The $5250 \AA$ line pair, however, does not measure $\mathrm{kG}$ fields in the cube (first row in Fig. 12). From this line pair, kG fields were not recovered either by Khomenko \& Collados (2007) in an MHD cube or by Socas-Navarro et al. (2008) in solar network observations. In the former paper, the authors explained this could be due to larger formation heights of the lines in the $5250 \AA$ pair and that they sampled weaker magnetic fields in the MHD cube. As $\mathrm{kG}$ fields could not be recovered in the network observations by Socas-Navarro et al. (2008), they concluded this line pair to be unreliable and that it is no better than the $6300 \AA$ pair in which the two lines are formed at very different heights in the atmosphere. This is surprising because the presence of $\mathrm{kG}$ fields in the solar network regions was discovered by applying MLR to the $5250 \AA$ line pair by Stenflo (1973).

To investigate this, we included a constant heightindependent $v_{\text {mac }}$ of $2 \mathrm{~km} \mathrm{~s}^{-1}$ in addition to $v_{\text {mic }}$ and recomputed the calibration curves. The $v_{\text {mic }}$ was varied to get the required line widths. Examples comparing the calibration curves with
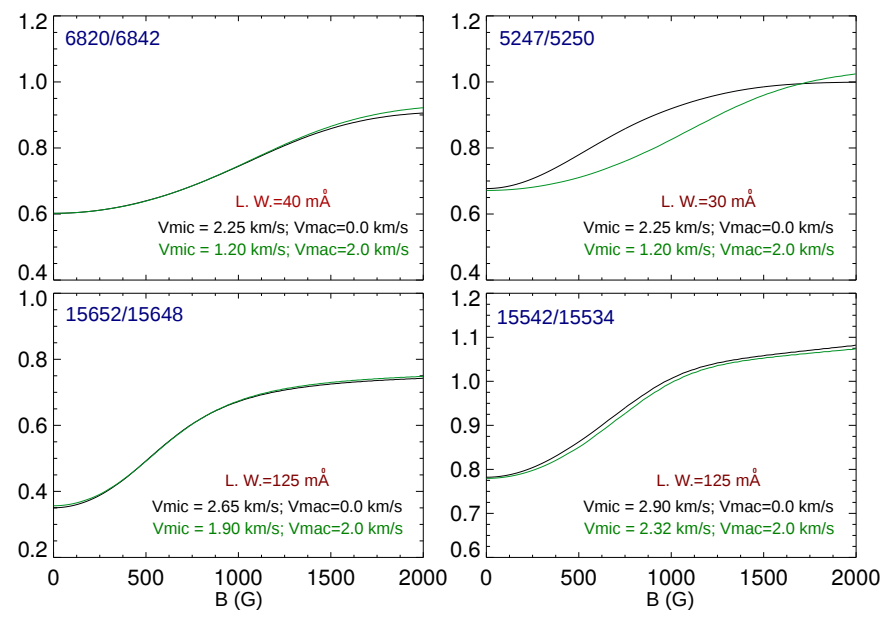

Fig. 11. Calibration curves for the four line pairs shown for sample line widths indicated by L.W. The black and the green curves represent line widths with and without a macro-turbulent velocity of $2 \mathrm{~km} \mathrm{~s}^{-1}$. In the green curves, the micro-turbulent velocity is reduced, to get the same line width.

and without $v_{\mathrm{mac}}$ for fixed line widths is shown in Fig. 11. The $5250 \AA$ line pair is the most affected by the addition of $v_{\text {mac }}$. This pair is highly sensitive to both $v_{\text {mic }}$ and $v_{\text {mac }}$, as pointed out in Solanki et al. (1987), Khomenko \& Collados (2007). The magnetic field strengths recovered from the new calibration curves are shown in the second row of Fig. 12. The $5250 \AA$ pair now shows the presence of $\mathrm{kG}$ fields in the cube. However, the magnetic field map from this line pair does not match well with those in the fourth row. This could be because of the approximations in the construction of the calibration curves. If the curves are constructed at every pixel by fitting the full spectral line then the $5250 \AA$ A pair may provide a better comparison with the magnetic field maps in the fourth row. The results from the other three line pairs are not much affected by the addition of $v_{\text {mac }}$, as also seen from Fig. 11. What we have presented is only a simplified approach, so that, if the $5250 \AA$ line pair is to be used for MLR, both $v_{\text {mic }}$ and $v_{\text {mac }}$ should be varied to match the line width and depth at every pixel in the cube. In any case, the 5250 line pair is less robust than the others.

\section{Conclusions}

The magnetic line ratio (MLR) method has been widely used to measure magnetic field strengths on the Sun. Until recently, three line pairs (5250 $\mathrm{A}, 6300 \AA$, and $1.56 \mu \mathrm{m}$ pairs) were used for this method, only two of which ( $5250 \AA$ and $1.56 \mu \mathrm{m}$ pairs) give reliable results. In this paper, we identified two new line pairs, the $6842 \AA$ pair in the visible and the $1.55 \mu \mathrm{m}$ pair in the IR. Lines in the $6842 \AA$ pair are separated by $22 \AA$ and those in the new $1.55 \mu \mathrm{m}$ pair by $8 \AA$. Lines in each of these pairs are formed at roughly the same height in the atmosphere. The new pairs have one line with high $\mathrm{g}_{\text {eff }}$ and with a large difference in $\mathrm{g}_{\text {eff }}$ between the lines, making them well suited for MLR. We presented a detailed comparison of the new and the old line pairs.

The Stokes profiles are synthesized in a three-dimensional MHD cube having a field strength $B_{\mathrm{MHD}}$, which differs from one pixel to the next. The MLR method is applied to the synthesized profiles to recover the field strengths, called $B_{\text {MLR }}$. The $B_{\text {MLR }}$ compares well when the $B_{\text {MHD }}$ is weighted with the Stokes $V$ response function and then integrated over the optical depth grid. 

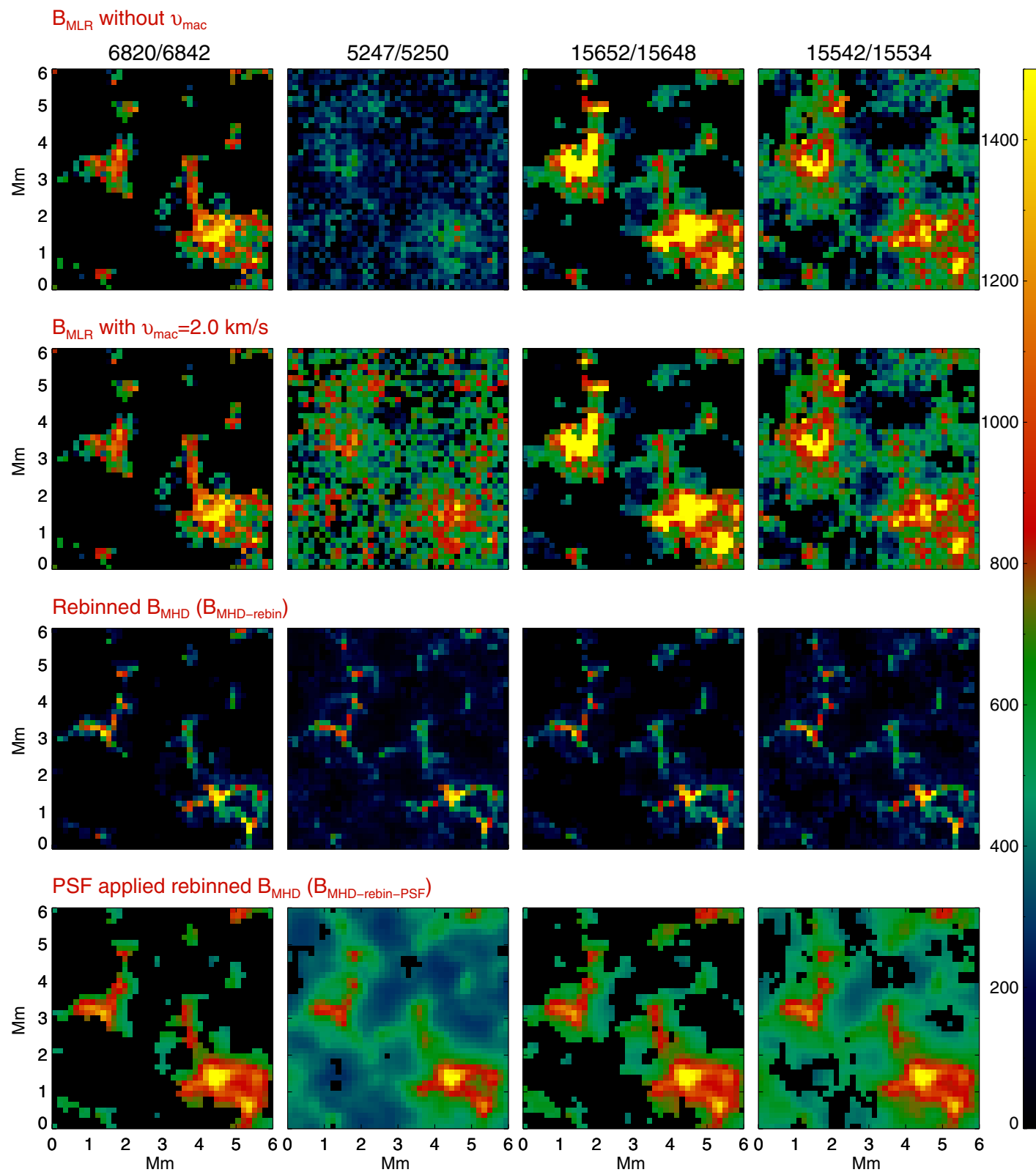

Fig. 12. $B_{\mathrm{MLR}}$ determined from spatially and spectrally degraded Stokes $V$ profiles affected by noise, using calibration curves without macroturbulent velocity (top row) and with a macro-turbulent velocity of $2.0 \mathrm{~km} \mathrm{~s}^{-1}$ (second row). This is compared with the magnetic field strength $B_{\text {MHD }}$ rebinned according to Eq. (3) ( $B_{\text {MHD-rebin }}$, third row). We show an empirical approximation of the result of MLR, based on $B_{\text {MHD-rebin }}$ and including the influence of the PSF in the fourth row. All profiles have been broadened with a micro-turbulence to match the widths of the profiles emerging from the MHD snapshot.

All the four line pairs reproduce $B_{\mathrm{MHD}}$, but the scatter in histogram of the difference between $B_{\mathrm{MHD}}$ and $B_{\mathrm{MLR}}$ is smaller for the new visible and IR pairs. The two lines in the new IR pair are stronger than the lines in the old $1.56 \mu \mathrm{m}$ pair. Although the lines in new IR pair have Stokes $V$ signals that are typically smaller than the $15648 \AA$ line $\left(g_{\text {eff }}=3\right.$ line), they are much stronger than those of the $\mathrm{g}_{\mathrm{eff}}=1.53$ line at $15652 \AA$ line, used together with $\lambda 15648 \AA$. Thus, in the presence of noise, the Stokes profiles of both lines in the new $1.55 \mu \mathrm{m}$ pair remain above noise more often than the $1.56 \mu \mathrm{m}$ pair, also making them favourable for inversions.

We further tested the line pairs by applying spatial and spectral degradation, and by adding random noise $\left(\sigma=1 \times 10^{-3} I_{\mathrm{c}}\right)$ to the Stokes profiles. We find that the new $6842 \AA$ pair and the old $1.56 \mu \mathrm{m}$ pair are most affected by noise. However, more than
$80 \%$ of the Stokes $V$ profiles from the new IR pair, remain above the $3 \sigma$ cut-off.

Using the $5250 \AA$ line pair, Khomenko \& Collados (2007) and Socas-Navarro et al. (2008) could not recover $\mathrm{kG}$ fields from the profiles synthesized in a 3D MHD cube and in the solar network observations, respectively. While Khomenko \& Collados (2007) attributed this to the larger formation heights of the lines in the $5250 \AA$ pair, Socas-Navarro et al. (2008) concluded this line pair to be unreliable. We find that the $5250 \AA$ pair is more sensitive to the nature of the velocity field, for example the exact mixture of micro and macro-turbulent velocities, than the other line pairs. Also, since the lines in this pair are strong and temperature sensitive, it is necessary to match the full line shape (line width and line depth) in the construction of calibration curves. From the calibration curves with the right combination 
H. N. Smitha and S. K. Solanki: Probing photospheric magnetic fields with new spectral line pairs

of micro and macro-turbulent velocities, it is possible to measure $\mathrm{kG}$ fields from the $5250 \AA$ pair. For the other three line pairs (6842 $\AA$, old $1.56 \mu \mathrm{m}$, and new $1.55 \mu \mathrm{m}$ pairs), calibration curves constructed by matching the line widths is sufficient for measuring reliable magnetic field strengths.

The interpretation of the MLR has in the past been generally given in terms of an idealized two-component atmosphere, a field free, and a homogeneous magnetic component (Stenflo 1973). In this representation the field strength returned by the MLR is an approximation of the intrinsic field strength in the magnetic component. What happens in a more realistic, complex atmosphere with a distribution of field strengths and the influence of a PSF? Here it turns out that the MLR still gives an approximation of the intrinsic field strength at the average formation height of the Stokes $V$ lobes, but weighted by the amplitude of the Stokes $V$ profile; regions with small Stokes $V$ provide a smaller contribution. Also the influence of spatial smearing turns out to be complex. Ours is the first attempt to determine empirically what exactly the MLR returns in a realistic atmosphere. It can likely be improved.

Sophisticated inversion codes are currently the preferred choice for magnetic field measurements. We expect the new line pairs to be attractive pairs for the application of inversion codes as well. In addition, it may be possible to combine the MLR with the inversions. One way would be to use the magnetic field strength measured from MLR as an initial guess in the inversions. Another is to employ the MLR as an additional constraint on the inversion. This will be investigated in a forthcoming paper.

Acknowledgements. We thank the referee for useful comments and suggestions that helped improve the paper. The authors are grateful to L. P. Chitta, A. Lagg, I. Milić, and M. van Noort for helpful discussions. Thanks to T. Riethmüller for kindly providing the MURaM MHD cube. H.N.S. acknowledges the financial support from the Alexander von Humboldt Foundation. This project has received funding from the European Research Council (ERC) under the European Union's Horizon 2020 research and innovation programme (grant agreement No. 695075) and has been supported by the BK21 plus programme through the National Research Foundation (NRF) funded by the Ministry of Education of Korea. This research has made use of NASA's Astrophysics Data System.

\section{References}

Balthasar, H., \& Schmidt, W. 1993, A\&A, 279, 243

Beckers, J. M., \& Milkey, R. W. 1975, Sol. Phys., 43, 289

Borrero, J. M., Lites, B. W., Lagg, A., Rezaei, R., \& Rempel, M. 2014, A\&A, 572, A54

Centeno, R., Socas-Navarro, H., Lites, B., et al. 2007, ApJ, 666, L137

Collados, M., López, R., Páez, E., et al. 2012, Astron. Nachr., 333, 872

de Wijn, A. G., Stenflo, J. O., Solanki, S. K., \& Tsuneta, S. 2009, Space Sci. Rev., 144,275

del Toro Iniesta, J. C. 2007, Introduction to Spectropolarimetry (Cambridge, UK: Cambridge University Press)

Domínguez Cerdeña, I., Kneer, F., \& Sánchez Almeida, J. 2003a, ApJ, 582, L55 Domínguez Cerdeña, I., Sánchez Almeida, J., \& Kneer, F. 2003b, A\&A, 407 741

Domínguez Cerdeña, I., Sánchez Almeida, J., \& Kneer, F. 2006, ApJ, 646, 1421
Elmore, D. F., Rimmele, T., Casini, R., et al. 2014, in Ground-based and Airborne Instrumentation for Astronomy V, Proc. SPIE, 9147, 914707

Frutiger, C. 2000, Ph.D. Thesis, Institute of Astronomy, ETH Zurich, No. 13896 Frutiger, C., Solanki, S. K., Fligge, M., \& Bruls, J. H. M. J. 2000, A\&A, 358, 1109

Gingerich, O., Noyes, R. W., Kalkofen, W., \& Cuny, Y. 1971, Sol. Phys., 18, 347

Grec, C., Uitenbroek, H., Faurobert, M., \& Aime, C. 2010, A\&A, 514, A91

Grossmann-Doerth, U., Schüssler, M., \& Steiner, O. 1998, A\&A, 337, 928

Ishikawa, R., \& Tsuneta, S. 2011, ApJ, 735, 74

Keller, C. U., Deubner, F.-L., Egger, U., Fleck, B., \& Povel, H. P. 1994, A\&A, 286, 626

Khomenko, E., \& Collados, M. 2007, ApJ, 659, 1726

Khomenko, E. V., Collados, M., Solanki, S. K., Lagg, A., \& Trujillo Bueno, J. 2003, A\&A, 408, 1115

Khomenko, E. V., Shelyag, S., Solanki, S. K., \& Vögler, A. 2005, A\&A, 442, 1059

Lagg, A., Solanki, S. K., Doerr, H.-P., et al. 2016, A\&A, 596, A6

Lin, H. 1995, ApJ, 446, 421

Lites, B. W., Kubo, M., Socas-Navarro, H., et al. 2008, ApJ, 672, 1237

Lozitsky, V. G., Lozitska, N. I., Lozitsky, V. V., et al. 1999, in Magnetic Fields and Solar Processes, eds. A. Wilson, et al., ESA SP, 448, 853

Martínez González, M. J., Collados, M., \& Ruiz Cobo, B. 2006, A\&A, 456, 1159

Martínez González, M. J., Collados, M., Ruiz Cobo, B., \& Solanki, S. K. 2007, A\&A, 469, L39

Martínez González, M. J., Collados, M., Ruiz Cobo, B., \& Beck, C. 2008, A\&A, 477,953

Ramsauer, J., Solanki, S. K., \& Biemont, E. 1995, A\&AS, 113, 71

Riethmüller, T. L., Solanki, S. K., Berdyugina, S. V., et al. 2014, A\&A, 568, A13

Rüedi, I., Solanki, S. K., Mathys, G., \& Saar, S. H. 1997, A\&A, 318, 429

Saar, S. H., Bünte, M., \& Solanki, S. K. 1994, in Cool Stars, Stellar Systems, and the Sun, ed. J.-P. Caillault, ASP Conf. Ser., 64, 474

Sánchez Almeida, J., Domínguez Cerdeña, I., \& Kneer, F. 2003, ApJ, 597, L177

Schmidt, W., von der Lühe, O., Volkmer, R., et al. 2012, Astron. Nachr., 333, 796

Schüssler, M., \& Solanki, S. K. 1988, A\&A, 192, 338

Shchukina, N., \& Trujillo Bueno, J. 2001, ApJ, 550, 970

Socas-Navarro, H., \& Sánchez Almeida, J. 2002, ApJ, 565, 1323

Socas-Navarro, H., \& Sánchez Almeida, J. 2003, ApJ, 593, 581

Socas-Navarro, H., Martínez Pillet, V., \& Lites, B. W. 2004, ApJ, 611, 1139

Socas-Navarro, H., Asensio Ramos, A., \& Manso Sainz, R. 2007, A\&A, 465, 339

Socas-Navarro, H., Borrero, J. M., Asensio Ramos, A., et al. 2008, ApJ, 674, 596

Solanki, S. K. 1993, Space Sci. Rev., 63, 1

Solanki, S. K. 2009, in Solar Polarization 5: In Honor of Jan Stenflo, eds. S. V. Berdyugina, K. N. Nagendra, \& R. Ramelli, ASP Conf. Ser., 405, 135

Solanki, S. K., \& Stenflo, J. O. 1985, A\&A, 148, 123

Solanki, S. K., Keller, C., \& Stenflo, J. O. 1987, A\&A, 188, 9

Solanki, S. K., Biemont, E., \& Muerset, U. 1990, A\&AS, 83, 307

Solanki, S. K., Rüedi, I. K., \& Livingston, W. 1992, A\&A, 263, 312

Solanki, S. K., Zufferey, D., Lin, H., Rüedi, I., \& Kuhn, J. R. 1996, A\&A, 310, L33

Steiner, O., \& Rezaei, R. 2012, in Fifth Hinode Science Meeting, eds. L. Golub, I. De Moortel, \& T. Shimizu, ASP Conf. Ser., 456, 3

Stenflo, J. O. 1973, Sol. Phys., 32, 41

Stenflo, J. O. 2010, A\&A, 517, A37

Stenflo, J. O. 2011, A\&A, 529, A42

Stenflo, J. O. 2013, A\&ARv, 21, 66

Stenflo, J. O., \& Harvey, J. W. 1985, Sol. Phys., 95, 99

Stenflo, J. O., Demidov, M. L., Bianda, M., \& Ramelli, R. 2013, A\&A, 556, A113

Vasilyeva, I. E., \& Shchukina, N. G. 2009, Kinematics and Physics of Celestial Bodies, 25, 319

Vögler, A., Shelyag, S., Schüssler, M., et al. 2005, A\&A, 429, 335

Wiehr, E. 2000, Sol. Phys., 197, 227 


\section{Appendix A: Anomalous Zeeman splitting and MLR}

In the presence of strong magnetic fields, the Zeeman saturation suppresses the amplitude of a Stokes $V$ profile and broadens its lobes. In normal Zeeman triplets, as $B$ increases, the amplitude of Stokes $V$ increases until it saturates. For field strengths beyond that, it remains constant. When the Zeeman splitting is anomalous, Stokes $V$ continues to change with $B$ (Solanki 1993). In the new line pairs, the $6820 \AA$ and $15534 \AA$ lines undergo anomalous Zeeman splitting. For stronger fields, their Stokes $V$ amplitudes decrease when $B$ exceeds a certain threshold value, $B_{\text {th }}$.

The $5247 \AA$ line is also not a normal Zeeman triplet, but its Stokes $V$ begins to decrease significantly only when $B$ exceeds $5 \mathrm{kG}$. A comparison between the $6820 \AA$ and $5247 \AA$ lines is shown in Fig. A.1. The behaviour of the Stokes $V$ amplitude is governed by the splitting of the individual transitions forming a given $\sigma$-component. For $B=1.5 \mathrm{kG}$, the separation between the various transitions in a $\sigma$ component $\left(\Delta \lambda_{B}\right)$ in the $6820 \AA$ line is as high as $22 \mathrm{~m} \AA$, whereas in the $5247 \AA$ line it is only $5 \mathrm{~m} \AA$ (indicated with red arrows in Fig. A.1). As $B$ increases, $\Delta \lambda_{B}$ becomes comparable to the line widths of the individual transitions, resulting in broadening of the $\sigma$ component and a corresponding decrease of the $V$ amplitude. This is clear when $B$ is increased to $4 \mathrm{kG}$, we see peaks of the line profiles from each transition in the $6820 \AA$ line (first column in Fig. A.1) but not in the $5247 \AA$ line (second column in Fig. A.1).
The $\Delta \lambda_{B}$ for a Zeeman component ( $\pi$ or $\sigma$ ) is proportional to $\left(m_{1} \mathrm{~g}_{1}-m_{\mathrm{u}} \mathrm{g}_{\mathrm{u}}\right)$, where $m_{\mathrm{l}, \mathrm{u}}$ are the magnetic quantum numbers of the lower and upper levels of the transition, respectively. The Landé g-factors of the upper and lower atomic levels are denoted as $\mathrm{g}_{\mathrm{u}}$ and $\mathrm{g}_{\mathrm{l}}$, respectively. For the $\sigma$ components, $\delta m=\left(m_{\mathrm{u}}-m_{1}\right)$ is \pm 1 and hence $\Delta \lambda_{B} \propto m_{\mathrm{u}}\left(\mathrm{g}_{1}-\mathrm{g}_{\mathrm{u}}\right) \pm \mathrm{g}_{1}$ (del Toro Iniesta 2007). For the $6820 \AA$ line, $\Delta \lambda_{B}$ is much larger with $\delta \mathrm{g}=\left|\mathrm{g}_{1}-\mathrm{g}_{\mathrm{u}}\right|=0.67$ and $\mathrm{g}_{1}=2.5$ compared to the $5247 \AA$ line with $\delta \mathrm{g}=0.25$ and $\mathrm{g}_{1}=0.5$. The $\Delta \lambda_{B}$ is large also for the $15534 \AA$ line with $\delta \mathrm{g}=0.33$ and $\mathrm{g}_{1}=1.5$. If $\mathrm{g}_{1}=0$ or $\mathrm{g}_{\mathrm{u}}=0$ or $\delta \mathrm{g}=0$ then it is a normal Zeeman triplet and there is no change in $V$ amplitude after Zeeman saturation. The calibration curves for MLR for line pairs with at least one line that undergoes anomalous Zeeman splitting do not saturate, that is, reach a constant value for stronger fields. Depending on whether the line with anomalous splitting is the magnetically weaker or the stronger in the pair, the calibration curve, when computed as the ratio of magnetically weaker to the stronger line, either decreases (6842 $\AA$ pair) or increases $(1.55 \mu \mathrm{m}$ pair) with $B$ as seen from Fig. 8.

The profiles in Fig. A.1 are computed without $v_{\text {mac }}$ and $v_{\text {mic }}$. Velocity broadening increases the value of $B_{\text {th }}$ at which Stokes $V$ starts to decrease (see Fig. 8). This behaviour, however, does not affect the diagnostic potential of the new line pairs as long as the line broadening is properly taken into account; this can easily be carried out by fitting the observed line profile.
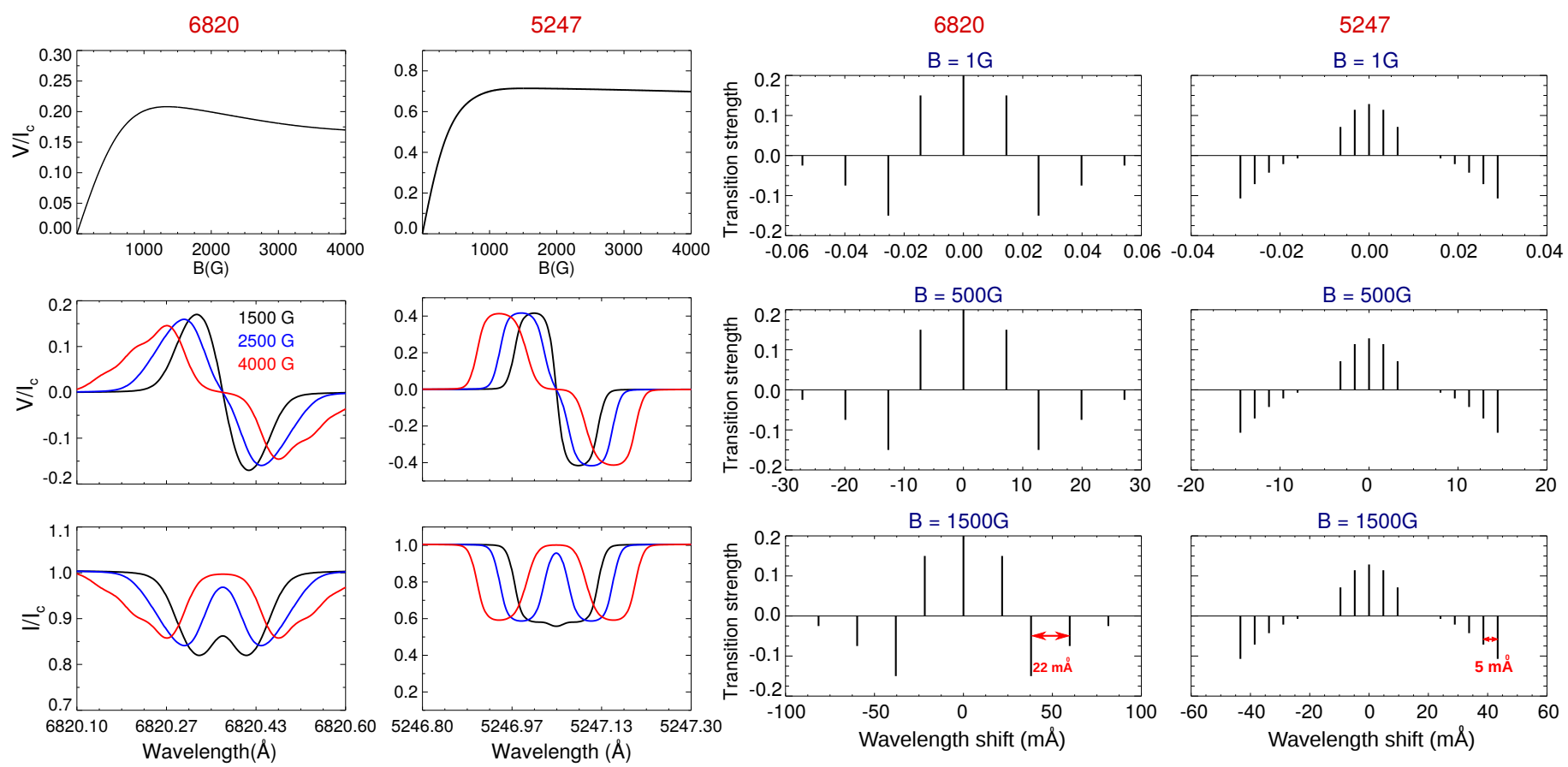

Fig. A.1. Variation in Stokes $V$ amplitude for the $6820 \AA$ and $5247 \AA$ lines as a function of magnetic field strength $(B)$. Full line profiles are shown for $1.5,2.5$, and $4 \mathrm{kG}$. A comparison between the Zeeman splitting pattern for the two lines for $B=1,500$ and $1500 \mathrm{G}$ is shown in the last two columns. There is a change in the wavelength scale of these plots with $B$. 